\title{
OCORRÊNCIA DE Lymnaea columella (SAY, 1817) (MOLLUSCA, PULMONATA, LYMNAEIDAE), NO ESTADO DE GOIÁS. CONSIDERAÇÕES SOBRE OS PARASITOS POR ELA TRANSMITIDOS.
}

\author{
José Luiz de Barros Araújo ", Guido Fontgalland Coelho Linhares"*, Julieta \\ Machado Paçố
}

\section{RESUMO}

Os autores relatam a primeira ocorrência de Lymnaea columella (Say, 1817) no Estado de Goiás, ressaltando a importância da espécie como hospedeiro intermediário de vários parasitos dos animais domésticos, dos quais, alguns são agentes de zoonoses no Brasil e em outros países. Também fazem consideraçð̃es sobre os parasitos transmitidos ou possivelmente transmitidos por este molusco, já assinalados no Brasil.

UNITERMOS: Lymnaea columella, ocorrência, ciclos evolutivos.

\section{INTRODUÇÃO}

Dentre os moluscos aquáticos da subordem Basommatophora, encontrados em água doce, no Brasil, Lymnaea columella (Say) é uma espécie de distribuição geográfica bastante ampla, constituindo-se o hospedeiro intermediário de vários

\footnotetext{
Prof. Titular do Depto. de Parasitologia do IPTSP-UFG.

** Prof. Adjunto do Depto. de Medicina Preventiva da Escola de Veterinária, Campus II, UFG

*** Prof. Adjunto do Depto. de Parasitologia do IPTSP-UFG.

Endereço para Correspondência:IPTSP-UFG. Cx. Postal, 131, CEP: 74001-970.

Trabalho financiado pelo CNPq, processo 520769/94.5

Recebido para publicação em 15/06/95
} 
ARAÚJO, J.L.B.; LINHARES, G.F.C.; \& PAÇÔ, J.M. Ocorrência de Lymnaea columella (Say, 1817) (Mollusca, Pulmonata, Lymnaeidae), no Estado de Goiás. Consideraçð̃es sobre os parasitos por ela transmitidos. Rev. Pat. Trop.24 ( 2): 291 - 300, jul/dez. 1995

parasitos de animais, embora não se conheçam em detalhes as relações molusco/parasito em todos os ciclos evolutivos que tenham a sua participação. Esta afirmativa tem base em estudos efetuados por pesquisadores em outros países, levando à suposição que tal fenômeno se repita no país.

Assim, ao avaliar sua participação no ciclo evolutivo dos parasitos, deparou-se com três situações importantes. A primeira é aquela em que esta participação é comprovada no país, tanto o parasito como seu hospedeiro intermediário têm suas ocorrências assinaladas. A segunda em que se relacionam os parasitos já identificados no país, e em outros países, e que, somente nestes comprovou-se participação. A terceira é aquela em que o molusco representa um hospedeiro potencial de parasitos ainda não assinalados no país, e que têm grandes repercussões nos aspectos sanitário e econômico, nas regiões onde ocorrem.

Na primeira situação, destaca-se a Fasciola hepatica, com distribuição em vários Estados do Brasil, provocando grandes perdas econômicas, principalmente para bovinos e ovinos, e também com relatos de casos de fasciolosis humana constituindo-se portanto em uma zoonose comprovada do parasito em algumas regiões endêmicas. Daí, o grande interesse no conhecimento da distribuição do parasito e de seus transmissores.

$\mathrm{Na}$ segunda situação, incluem-se várias espécies da família Paramphistomatidae, que podem realizar seu ciclo evolutivo em Lymnaea columella. A literatura refere freqüentemente a Lymnaea sp como hospedeiro intermediário de

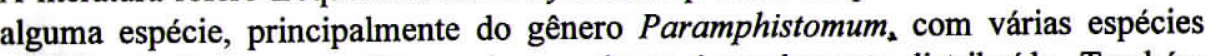
sendo assinaladas em regiões onde o molusco é amplamente distribuído. Também destaca-se a possibilidade de atuação deste molusco no ciclo evolutivo de Echinostoma revolutum de aves, abrigando as metacercárias. Este trematódeo é considerado um antagonista de Schistosoma mansoni em sua fase evolutiva em Biomphalaria.

$\mathrm{Na}$ terceira situação encontra-se a participação no ciclo evolutivo de espécies como Heterobilharzia americana de cães, coelho, lince, guaxinim (MALEK, 1974), Angiostronglus cantonensis da artéria pulmonar e às vezes do ventrículo direito do rato, e acidentalmente do homem (MALEK, 1974), além de outros, e que, ainda não foram assinalados no Brasil. Devem-se mencionar também a referência a Fasciola gigantica feita por COSTA e Cols. (1986), com base em citaçðes de PINTO (1945). Estes autores baseiam-se em VIANA (1934), assinalando o parasitismo nos canais biliares de ovinos, com material sem registro de procedência. PINTO (1945) faz referência ao achado de HASSELMANN (1915) no apéndice do homem no Rio de Janeiro. Refere também que o parasitismo ocorre no figado (vias biliares) de cabras e algumas vezes nos intestinos do homem, bovino, ovino, caprino, burro e porco doméstico.
ARAÚJO, J.L.B.; LINHARES, G.F.C.; \& PAÇÔ, J.M. Ocorrência de Lymnaea columella (Say, 1817) (Mollusca, Pulmonata, Lymnaeidae), no Estado de Goiás. Consideraçð̃es sobre os parasitos por ela transmitidos. Rev. Pat. Trop.24 ( 2 ): 291 - 300, jul/dez. 1995

\section{LITERATURA CONSULTADA}

LUTZ (1921) assinala a ocorrência de Fasciola hepatica, no Estado do Rio de Janeiro, sem contudo identificar com segurança a espécie capaz de atuar como hospedeiro intermediário.

HARRY e HUBENDICK (1964), fazem uma descrição detalhada não só da concha, como também das partes moles de Lymnaea columella (Say, 1817) e Lymnaea cubensis (Pfeiffer, 1839). Caracterizam também a família Lymnaeidae e o gênero Lymnaea.

VAN EEDEN, e cols.(1965) em trabalho sobre os moluscos de água doce de importância médica humana e veterinária na África do Sul, discute aspectos importantes ligados às condições ambientais, que influenciam tanto os parasitos como seus transmissores. Também faz referência à Lymnaea columella e os parasitos a ela relacionados. Em sua discussão faz referência à Lymnaea columella como uma espécie de importância econômica como hospedeiro intermediário de Fasciola spp.

REZENDE e cols. (1973), além de assinalarem pela primeira vez no Brasil a presença de Lymnaea cubensis. PFEIFER (1839), fizeram um estudo comparativo da evolução de Fasciola hepatica, nesta espécie e em Lymnaea columella.

MALEK e CHENG (1974), além de identificarem sua posição sistemática, considerando-a Pseudosuccinea columella, destacam sua participação como hospedeiro intermediário de alguns parasitos, dentre eles Fasciola hepatica e Fasciola gigantica.

COSTA e Cols. (1974) em infecção experimental confirmam a participação de $L$. columella no ciclo evolutivo de Fasciola hepatica, no Estado do Rio de Janeiro.

GOMES e Cols (1974) através de infecção experimental comprovaram a participação de Lymnaea columella no ciclo de Fasciola hepatica, de ocorrência no Estado do Rio de Janeiro.

DACAL e Cols (1988) avaliaram em trabalhos experimentais a susceptibilidade de estirpes de Lymnaea columella de diferentes procedências à Fasciola hepatica, não constatando diferenças. Concluíram também que as populações humanas, bovina, ovina e suína de Minas Gerais, podem ser consideradas sujeitas à infecção por Fasciola hepatica.

\section{MATERIAL E MÉTODOS}

Os moluscos, num total de 13 exemplares foram colhidos em córregos e valas irrigadas, presos a diferentes substratos, onde foram encontradas também 
ARAÚJO, J.L.B.; LINHARES, G.F.C.; \& PAÇÔ, J.M. Ocorrência de Lymnaea columella (Say, 1817) (Mollusca, Pulmonata, Lymnaeidae), no Estado de Goiás. Consideraçð̃es sobre os parasitos por ela transmitidos. Rev. Pat. Trop.24 ( 2 ): 291 - 300, jul/dez. 1995

algumas posturas. Nestes mesmos locais foram colhidos alguns exemplares de Physa $\mathrm{p}$, Biomphalaria straminea, Thiara sp, além de alguns Bivalvia, situados em pontos diferentes do município de Goiânia, Estado de Goiás, nos meses de junho e julho de 1994. Todos os exemplares de $L$. columella examinados encontravam-se negativos à infecção por parasitos, fato comprovado durante as dissecações.

Os moluscos foram mantidos em líquido de Railliet-Henry. Aqueles destinados às dissecações foram primeiramente retirados das conchas após aquecimento em água a $60^{\circ} \mathrm{C}$ e dissecados em microscópio estereoscópio no mesmo líquido, separando-se os sistemas digestivo e genital, o bulbo da rádula e a câmara palial, que, é a parte que mais segurança oferece para identificação da espécie, separando-a de $L$. cubensis, (Pfeiffer) que também já foi assinalada no país, foi desenhada juntamente com a concha, ao microscópio estereoscópiò equipado com câmara clara.

O sistema genítal foi desidratado na série crescente de álcoois, corado com carmim clorídrico e clarificado em creosoto, onde foi estudado.

$\mathrm{O}$ bulbo da rádula foi fervido em potassa a $10 \%$, separando-se a rádula e mandíbula, que foram mantidas em líquidos de Railliet-Henry para posteriores montagens.

\section{RESULTADOS}

Lymnaea columella (Say, 1817)

Sinonímia $\quad$ - Pseudosuccinea columella, Malek e Cheng, 1974: 55-66.

- Lymnaea (Pseudosuccinea) columella, Thiele, 1931: 476.

- Lymnaea columella, Harry e Hubendick, 1964: 8-10, figs. 69 e 109.

CONCHA. As conchas são dextrogiras, medindo $10,5 \mathrm{~mm}$ de comprimento e $5,5 \mathrm{~mm}$ de largura, de coloração amarelo testáceo, uniformemente colorida, com voltas de espira, suturas bem evidentes, de consistência delgada, volta corporal correspondendo à maior parte do comprimento da concha. Abertura ovalada, com lábio externo cortante, sem qualquer formação semelhante a dentes ou lamelas. Concha embrionária, sem esculturas, sem marcadores limites das voltas seguintes.

PARTES MOLES. No estudo da anatomia visceral, destaca-se principalmente o aspecto apresentado pela câmara palial, com relação à disposiçăo do ureter secundário, que apresenta duas flexuras, diferenciando-a da espécie mais próxima e já assinalada no Brasil, a Lymnaea cubensis (Pfeifer, 1839).
ARAÚJO, J.L.B.; LINHARES, G.F.C; \& PAĈ, J.M. Ocorrência de Lymnaea columella (Say, 1817) (Mollusca, Pulmonata, Lymnaeidae), no Estado de Goiás. Consideraçðes sobre os parasitos por ela transmitidos. Rev. Pat. Trop.24 ( 2): $291-300$, jul/dez. 1995

A distribuição geográfica de Lymnaea columella por unidade da Federação, dos parasitos nos quais está envolvida em seu ciclo e dos hospedeiros definitivos destes parasitos estão demonstrados no quadro abaixo:

Quadro 1. Distribuição geográfica de Lymnaea columella e dos parasitos e hospedeiros, a ela relacionados.

\begin{tabular}{|c|c|c|c|c|c|c|c|c|c|c|c|c|c|c|c|}
\hline & \multicolumn{14}{|c|}{ HOSPEDEIROS } \\
\hline & & L. columella & Hom. & Bov. & Ovi. & Cap. & $\begin{array}{l}\text { Bub. } \\
\text {. }\end{array}$ & Sui. & Can. & Equ. & Gal. & Pat. & Pom. & Rat. & Coe. \\
\hline & Amapá & - & $\cdots$ & -+- & $\cdots$ & $\cdots$ & $\cdots$ & $\cdots$ & $\cdots$ & $\cdots$ & $\cdots$ & $\cdots$ & $\cdots$ & $\cdots$ & $\cdots$ \\
\hline D & Cearín & - & $\cdots$ & $\cdots$ & -+- & $\cdots$ & $\cdots$ & $\cdots$ & $\cdots$ & $\cdots$ & $\cdots$ & $\cdots$ & $\cdots$ & $\cdots$ & $\cdots$ \\
\hline $\mathbf{I}$ & Bahia & - & +-- & $\cdots$ & $\cdots$ & $\cdots$ & $\cdots$ & $\cdots+$ & $\cdots$ & $\cdots$ & $\cdots$ & $\cdots$ & $\cdots$ & $\cdots$ & $\cdots$ \\
\hline $\mathbf{s}$ & Goiks & + & $\cdots$ & ++- & $\cdots$ & $\cdots$ & $\cdots$ & $\cdots$ & $\cdots$ & $\cdots$ & $\cdots$ & $\cdots$ & $\cdots$ & $\cdots$ & $\cdots$ \\
\hline $\mathbf{T}$ & Mt.Grosso & - &.+- & $\cdots$ & $\cdots$ & $\cdots$ & $\cdots$ & $\cdots$ & $\cdots$ & $\cdots$ & $\cdots$ & $\cdots$ & $\cdots$ & $\cdots$ & $\cdots$ \\
\hline $\mathbf{R}$ & Brasil Central & - & $\cdots$ & $\cdots$ & $\cdots$ & $\cdots$ & $\cdots$ & $+\cdots$ & $\cdots$ & $\cdots$ & $\cdots$ & $\cdots$ & $\cdots$ & $\cdots$ & $\cdots$ \\
\hline I & R.J. & + &.+- & $(+)--$ & $+\ldots$ & $\cdots$ & $\cdots$ & $\cdots$ & $\cdots$ & $\cdots$ & $\cdots+$ & $\cdots$ & $\cdots+t^{n}$ & $\because(+)$ & $\cdots$ \\
\hline B & S. Paulo & + & $+\cdots$ & $(+)--$ & $+\cdots$ & $\cdots$ & $\cdots$ & $+\ldots$ & $\cdots$ & $\cdots$ & $\cdots$ & $\cdots$ & $\cdots$ & $\cdots$ & $\cdots$ \\
\hline U & M.Gerais & + & -. & $(+)-$ & $\cdots$ & $\cdots$ & $\cdots$ & $\cdots$ & $\cdots$ & $\cdots$ & $\cdots$ & $\cdots+$ & $\cdots$ & $\cdots$ & $\cdots$ \\
\hline I & Paraná & + & $+\cdots$ & $(+)--$ & $+\cdots$ & $+\cdots$ & $+\ldots$ & $+\ldots$ & $+\cdot t^{n}$ & $+\cdots$ & $\cdots$ & $\cdots$ & $\cdots$ & $\cdots$ & $\cdots$ \\
\hline ç & \begin{tabular}{|l} 
Sta.Catarina \\
\end{tabular} & + & $\cdots$ & $(+)^{+-}$ & $\cdots$ & $\cdots$ & $\cdots$ & $\cdots$ & $\cdots$ & $\cdots$ & $\cdots$ & $\cdots$ & $\cdots$ & $\cdots$ & $\cdots$ \\
\hline$\overline{\mathbf{A}}$ & RG. do Sul & + & $+-t$ & $(+)^{++-}$ & ++- & $\cdots$ & -+- & $\cdots$ & $\cdots$ & $\cdots$ & $\cdots$ & $\cdots$ & $\cdots$ & $\cdots$ & $\cdots$ \\
\hline & Outros paises & + & +-+ & $\frac{(++)^{+-}}{4}$ & ++- & $\cdots$ & -+- & $\cdots$ & $\cdots$ & $\cdots$ & $\cdots$ & $\cdots$ & $\cdots$ & $\cdots$ & $+\cdots$ \\
\hline & & & $\overline{\text { FPE }}$ & FPE & FPE & FPE & FPE & FPE & $\overline{\text { FPE }}$ & FPE & FPE & FPE & FPE & FPE & FPE \\
\hline
\end{tabular}

$\mathrm{F}=$ Fasciola hepatica $; \mathrm{P}=$ Paramphistomum spp $\mathrm{E}=$ Echinostoma revolutum; $+=$ Parasito presente; $(+)=$ Parasito presente, com ciclo conhecido;

-= Parasito ausente; " = Possivelmente Echinostoma revolutum Ho = homem; $\mathrm{Can}=$ caninos; $\mathrm{Bov}=$ bovino; $\mathrm{Equ}=$ equinos; $\quad$ Ovi = ovino, Gal = galinha; Cap = caprino; Pat $=$ Pato; $\mathrm{Bub}=$ bubalinos; Rat = rato; Sui = Suínos; Coe $=$ coelho. 
ARAÚJO, J.L.B.; LINHARES, G.F.C.; \& PAÇÔ, J.M. Ocorrência de Lymnaea columella (Say, 1817) (Mollusca, Pulmonata, Lymnaeidae), no Estado de Goiás. Considerações sobre os parasitos por ela transmitidos. Rev. Pat. Trop.24 ( 2): 291 - 300, jul/dez. 1995

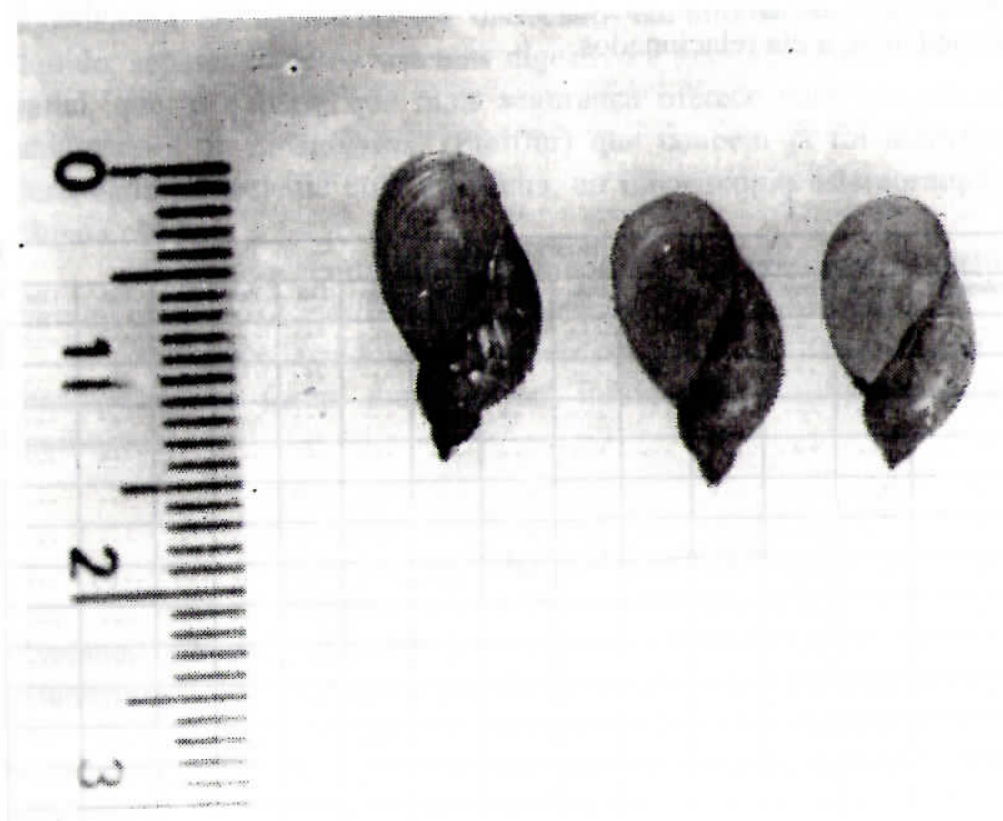

Figura 1. Conchas de Lymnaea columella

\section{DISCUSSÃO E CONCLUSÕES}

A presença deste molusco no Estado de Goiás representa a possibilidade de ocorrência de vários parasitos de animais e do homem, que dele se servem como hospedeiro intermediário. Estes parasitos merecem atenção, não só pela sua patogenicidade para as espécies em que ocorrem, como também pelas perdas econômicas que determinam sobre tais espécies. Assim, o fato de poucas espécies terem sido assinaladas no Estado de Goiás, não exclui a importância do achado de
ARAÚJO, J.L.B.; LINHARES, G.F.C.; \& PAÇÔ, J.M. Ocorrência de Lymnaea columella (Say, 1817) (Mollusca, Pulmonata, Lymnaeidae), no Estado de Goiás. Consideraçð̃es sobre os parasitos por ela transmitidos. Rev. Pat. Trop.24 ( 2 ): 291 - 300, jul/dez. 1995

um transmissor que, em outras regiões do Brasil, merecem severas medidas de controle. Recentemente ARAÚJO e Cols. identificaram casos de Fasciolosis hepática em bovinos abatidos em Goiânia, que foram introduzidos no Município de Santa Helena para engorda, trazidos do Rio Grande do Sul. Se tal fato coincidiu com a ocorrência do molusco na região, as possibilidades de instalação de um foco são grandes, desde que as demais condições tenham existido. Por outro lado, as descargas de matadouros são sempre dirigidas para rios e córregos onde prolifera o molusco, o que coincidiu com a região de abate dos animais.

Até o momento, embora tenham sido colhidos apenas alguns exemplares de Paramphistomatídeos, ainda não identificados, não se constatou na literatura nenhum registro, para o Estado de Goiás já que COSTA (1986) cita SIMÕES (1974) que se referiu em um trabalho, a um foco de Amphistomose bovina no norte de Goiás, região que atualmente pertence ao Estado do Tocantins. Alguns exemplares foram colhidos no Estado demonstrando a presença em bovinos nascidos e criados na região.

Quanto à Fasciola gigantica referida por COSTA (1986), verifica-se que os casos de parasitismo no Brasil ficaram restritos àquelas citações, não sendo referido em datas posteriores, o que induz a aguardar estudos mais recentes para confirmar sua presença no país, pois, segundo MALEK (1974), L. columella é seu hospedeiro intermediário.

Outros parasitos que merecem atenção são pertencentes à família Echinostomatidae, na qual se destaca o gênero Echinostoma, em que várias espécies podem parasitar animais domésticos e silvestres, e também o homem.

No ciclo evolutivo destes parasitos, a fase até a formação de cercárias dá-se em moluscos aquáticos do gênero Biomphalaria que, segundo PARAENSE (1975), várias espécies ocorrem em Goiás. As metacercárias podem encistarem-se em moluscos como Physa sp, Biomphalaria Lymnaea, etc, além de outros organismos aquáticos. Raras são as citações de echinostomatídeos em Goiás. Destacam-se apenas os trabalhos de KOMMA (1972) sobre a revalidação de Echinostoma erraticum Lutz, 1924 e sobre os aspectos morfológicos e biológicos da mesma espécie (KOMMA, 1974). Esta espécie é citada por TRAVASSOS e Cols (1969) como sinônima de Echinostoma revolutum e parasitando intestino de galinha e pato, cloaca e bolsa de Fabricius de pato. Levando-se em conta o conceito de TRAVASSOS, acrescenta-se também o rato (BARBOSA, 1991). Salienta-se a importância dos Echinostomatídeos como parasito que pode eventualmente atacar o homem (ACHA, 1986), como também se considera que algumas formas intermediárias de algumas espécies podem atuar como antagonistas do Schistosoma mansoni.

Isto posto, considera-se que a presença do molusco em Goiás, Estado com pecuária forte, deva merecer especial atenção, particularmente nas regiões onde se 
ARAÚJO, J.L.B.; LINHARES, G.F.C.; \& PAÇÔ, J.M. Ocorrência de Lymnaea columella (Say, 1817) (Mollusca, Pulmonata, Lymnaeidae), no Estado de Goiás. Consideraçס̃es sobre os parasitos por ela Mransmitidos. Rev. Pat. Trop.24 (2 ): 291 - 300, jul/dez. 1995

comprovar a ocorrência de algum parasito que dele necessite para realizar o seu ciclo. Para o homem, nas áreas de grandes concentrações humanas populacionais, como nas cidades, os cuidados relativos à Saúde Pública devem ser permanentes, evitando-se que os fatores favoráveis ao desenvolvimento do ciclo evolutivo dos parasitos se completem.

\section{AGRADECIMENTOS}

Externamos nossos agradecimentos aos bolsistas do CNPq Jefferson Borges de Andrade, Hélvio Queiroz dos Santos e Cláudia Regina Ferreira da Silva (Processo CNPq 520769/94-5, (N.V), pela participação nos trabalhos de colheita e preparação do material estudado e ao Laboratorista Edimar Luiz Alves.

\section{SUMMARY}

Occurrence of Lymnaea columella (Say, 1817) (Mollusca, Pulmonata, Lymnaeidae) in the State of Goiás and comments about the relationships of the mollusc with the transmission of some parasites

The authors report the first occurrence of Lymnaea columella in the State of Goiás, bringing out its relevance as the intermediate host of parasites from several domestic animals. Also considerations are made on the parasites already identified in Brazil, transmitted or possibly transmitted by this mollusc.

KEYWORDS: Lymnaea columella, occurrence, life cycle.

\section{REFERÊNCIAS BIBLIOGRÁFICAS}

ACHA, P.N. e SZYTRES, B. ZOONOSIS, Y. Enfermedades transmisibles comunes al hombre y a los animales. Segunda Edicion. Org. Pan-Anamericana de la salud: 672-674. 1986.

ARAÚJO, J.L. de B., GARCIA, C.A., LINHARES, G.F.C. Informação pessoal. 1994.

BARBOSA, J.V. Infecção natural e formas evolutivas de Echinostoma revolutum (Froelich, 1802) em Biomphalaria tenagophila ( Orbigny, 1835) e Rattus
ARAÚJO, J.L.B.; LINHARES, G.F.C.; \& PAÇÔ, J.M. Ocorrência de Lymnaea columella (Say, 1817) (Mollusca, Pulmonata, Lymnaeidae), no Estado de Goiás. Considerações sobre os parasitos por ela

transmitidos. Rev. Pat. Trop.24 ( 2 ): 291 - 300, jul/dez. 1995

norvegicus. Susceptibilidade de Mus musculus ao parasitismo. Tese. Mimeografado. 60p. 1991.

BONNE, C., BRAS, G. e LIE, K.L. Five Echinostomes found in man in the Malayan Archipelago. American Journal of Disease 20: 12-16.

C.T. e CROSSS, J.H. Observation on the nostparasite relations between Echinostoma revolutum and Lymnaeid snails. Chiw. J. Microbiol. 8: 241-2551. 1975.

CARVALHO, O.S., KAWAZAE, e CUBA, C.C.A. Echinostoma revolutum (Froelich, 1802) (Trematoda, Echinostomatidae) em pato doméstico em Minas Gerais. Atas Soc. Biol. Rio de Janeiro 17(2): 73-75. 1974.

DACAL, A.R.C., COSTA, H.M.A. e LEITE, A.C.R. Susceptibilidade de Lymnaea (Pseudosuccinea) columella (Say, 1817) exposta à infecção por miracídeos de Fasciola hepatica, (Linnaeus, 1758). Rev. Inst. Med. Trop. São Paulo 30(5): 361-369, 1988.

GOMES, P.A.C., NUERNBERG, S., PIMENTEL NETO, M., OLIVEIRA, G.P.; RESENDE, H.E.B., ARAÚJO, J.L. de B., e, MELO, R.P. Infecção experimental de Lymnaea columella Say, 1817, com Fasciola hepatica, Limnnaeus, 1758, de ocorrência no Estado do Rio de Janeiro. Arq. Univ. Fed. Rur. Rio de Janeiro 4(1): 35-38. 1974.

HARRY, H.W. e HUDENDICK, B. the freshwater pulmonate mollusca of Puerto Rico. Goteborgs VetenskSamh. Hande. Ser B Band 9, n. 5: 1-77. 1963.

KOHN, A. e BUSHNHEIN, V. Infestação natural de Echinostoma revolutum (Froelich, 1802) (Trematoda, Echinostomatidae) em porco doméstico. Atas Soc. Biol. Rio de Janeiro 16(1): 5-6.

KOMMA, M.D. Revalidação da espécie Echinostoma erraticum Lent, 1924. Rev. Pat. Trop. 1(4): 463-471. 1972.

KOMMA, M.D. Trematódeos digenéticos (Echinostomatidae) do Estado de Goiás Brasil. Aspectos morfológicos e biológicos do esporocisto de Echinostoma erraticum Lentz, 1924 (Mendheim, 1940) Konina, 1972. Rev. Pato. Trop. 3(1): 57-63. 1974. 
ARAÚJO, J.L.B.; LINHARES, G.F.C.; \& PAÇÔ, J.M. Ocorrência de Lymnaea columella (Say, 1817) (Mollusca, Pulmonata, Lymnaeidae), no Estado de Goiás. Considerações sobre os parasitos por ela transmitidos. Rev. Pat. Trop.24 ( 2 ): 291 - 300, jul/dez. 1995

MALEK, E.A. e CHENG, T.C. Medical and Economic Malacology 398pags. Academic Press Ed. 1974.

MELO, A. Da ocorrência de Echinostoma revolutum_ (Froelich, 1802) em Gallus domesticus no Brasil. Arch Esc, Sup. Agric. Med. Vet. Rio de Janeiro 10(2): 172. 1933.

PARANAENSE, W.L. Estado atual da sistematica dos planorbídeos brasileiros (Mollusca, Gastropoda). Arq. Mus. Nac. 55: 105-127. 1975

PINTO, C. Zooparasitos de interesse médico e veterinário. 375p. Pimenta de Melo Ed. 1945.

REZENDE, H.B.R., ARAÚJO, J.L. de B., GOMES, P.A.C., NUERNBERG, S., PIMENTELS NETO, M., OLIVEIRA, G.P. e MELO, R.P. Notas sobre duas espécies de Lymnaea LAMARCK, 1799, hospedeiros intermediários de Fasciola hepatica, L. no Estado do Rio de Janeiro (Mollusca, Gastropoda, Basommatophora, Lymnaeidae). Arq. Univ. Fed. Rur. Rio de Janeiro 3(1): 2123. 1973.

SOULSBY, E.J.L. Textbook of Veterinary Clinical Parasitology e Helminths 1120p. Blackwell Scientific Publications. Ed. 1965.

THIELE, J. Handbuch der Systematischum Weichtierkunde 778p. Verlag von Gustav Fischer Ed. 1931.

TRAVASSOS, L., TEIXEIRA DE FREITAS, J.F. e HKOHN, A. Trematódeos do Brasil. Mem. Inst. Oswaldo Cruz 67: 1-886. 1969.

VAN EeDEN, J.A., BROWN, D.S. e OBERHOLZER, G. The distribuiton of freshwateer mollusc of medical and veterinary importance in south-eastern. Africa. Ann. Trop. Med. Parasit. 59(4): 413-424. 1965.

VELAZQUEZ-MALDONADO, J.J. Estudo taxonômico dos trematódeos paramphistomiformes do rumen de bovinos do Estado do Rio Grande do Sul. Brasil. 86p. Fundação Cargill Ed. 1976. 